\title{
Transplantation and tolerance
}

\author{
David Perkins
}

Published online: 15 April 2011

(C) Springer-Verlag 2011

Solid organ transplantation is the treatment of choice for many end-stage organ diseases and can be life saving for patients with end-stage lung, liver and heart diseases. The development of immunosuppressive agents including calcineurin inhibitors and induction therapy with antibodies has markedly decreased the incidence of acute rejection. However, long-term graft survival has not correspondingly improved. Since the strongest predictor of chronic rejection was the occurrence of acute rejection, the lack of improvement in long-term graft survival was unexpected. Thus, improvements in outcomes in clinical transplantation will require novel therapeutic strategies. One approach is the development of clinical tolerance, which is the focus of this edition and remains the "holy grail" in transplantation research. The definition of tolerance is commonly considered to be the lack of rejection in the absence of immunosuppressive treatment. However, based on mechanistic studies in model systems, it is apparent that tolerance is complex and involves multiple mechanisms. Furthermore, the incidence of graft rejection varies markedly among different organs. For example, lung transplant recipients have the worst outcomes of all solid organ recipients, whereas approximately $20 \%$ of all liver transplant recipients can be successfully weaned from immunosuppressive drugs. Based on these considerations, this edition will include reviews that discuss clinical tolerance in kidney, lung and liver transplantation. In addition, reviews of the various immune components and cellular subsets that contribute to the rejection process are included.

D. Perkins $(\square)$

Transplantation Research, Division of Nephrology,

Department of Medicine, University of California San Diego,

San Diego, CA, USA

e-mail: davperkins@ucsd.edu
As reviewed by K. Newell in "Clinical Transplantation Tolerance", investigations of clinical tolerance are challenging due to the lack of established protocols to induce tolerance and lack of established biomarkers to detect and define tolerance. Thus, much of our current mechanistic understanding is derived from animal models and in vitro studies. However, spontaneous cases of clinical tolerance are occasionally observed in kidney transplant patients that maintained normal graft function and histology despite discontinuing all immunosuppression, usually due to noncompliance with immunosuppressive medications. In a study of 25 patients sponsored by the Immune Tolerance Network, 30 differentially expressed genes were identified in peripheral blood mononuclear cells. In another study sponsored by the European Union, an increased frequency of less mature B cells was observed in the tolerant subjects. Thus, B cells may have a currently poorly understood role in maintaining clinical tolerance. Several interventional studies of protocols to induce tolerance are currently in process. One promising result by Fudaba et al. involved non-myeloablative bone marrow transplant and renal transplant in six patients with excellent long-term renal function. Also, studies currently in progress are discussed.

Since spontaneous clinical tolerance is a rare event, it is imperative that we investigate the mechanisms that prevent tolerance. W. Burlingham in "Loss of Tolerance to Self after Transplant" discusses recent findings that chronic rejection, which is a major mode of graft loss, is associated with the loss of self-tolerance and promoted by IL-17dependent autoimmunity. The exposure of "cryptic selfantigens" has been both heart (cardiac myosin and vimentin) and lung (collagen type $\mathrm{V}$ and $\mathrm{K}-\alpha 1$ tubulin) transplantation. The most convincing results have been obtained in lung transplant models involving human, rat and mouse studies. These studies suggest that chronic 
rejection involves a critical role for an autoimmune process that targets autoantigens by Th17 responses.

To develop strategies to induce tolerance, it is crucial to understand the mechanisms of alloimmunity. Several areas of research have proved fertile in increasing our understanding of graft rejection. As reviewed by D. Goldstein in "Inflammation and Transplantation Tolerance", innate immune signals may modulate susceptibility to tolerance. For example, one explanation for differential tolerance susceptibility is that tolerant-resistant organs (e.g. skin) activate stronger innate immune signals than tolerant-susceptible organs (e.g. liver). Susceptibility to tolerance may be regulated, at least in part, by inflammatory cytokines including IL-6, TNF- $\alpha$ and type I interferons. As reviewed by D. McKay in "Role of Innate Immunity in Donor Organ Procurement", injury of the donor organ is mediated by innate immune receptors including tolllike receptors, nucleotide-binding oligomerization domainlike receptors and retinoic acid-inducible gene-I-like receptors. Understanding innate immune injury is increasingly important as extended criteria donors and deceased cardiac death donors are being utilized. Important insults, including ischaemia, reperfusion and brain death, are presumably mediated via damage-activated molecular patterns that include a panel of endogenous ligands. These observations suggest the importance of evaluating the quality of donor grafts. As reviewed by T. Mueller in "Assessment of Donor Organ Quality and Prediction of Outcome at Time of Transplantation", the evaluation of donor grafts is currently performed by histological analysis of biopsy specimens. However, histopathology, although important, has wellknown limitations. Recent studies using gene profiling of preimplantation graft biopsy tissue indicate that molecular profiles document changes not detected by morphology or clinical markers. Importantly, molecular profiles can provide a quantitative assessment of inflammatory and immune responses that reflect pathways associated with injury and repair. A proposed approach to predict graft outcomes is based on an integration of clinical and molecular parameters.

An understanding of the mechanisms of innate immune injury to the donor graft suggests novel therapeutic targets to improve graft outcomes. Current therapeutic strategies focus on inhibition of the adaptive immune response with the partial exception of steroids. By inhibiting NF- $\mathrm{KB}$, steroids can inhibit components of the innate, as well as the adaptive, responses. As reviewed by R. Steiner in "Steroids in Kidney Transplant Patients", corticosteroids have numerous nonspecific anti-inflammatory properties that prevent rejection after organ transplantation. Due to the side effects of steroid administration, tapering and steroid-free protocols have been attempted; however, substantial therapeutic benefits are also apparent. A thorough discussion of risk versus benefit has been presented. As reviewed by P. Naik in "Special Issues in the Management and Selection of the Donor for Lung Transplantation", another strategy to deal with the problem of donor graft injury and to increase the donor pool is to thoroughly evaluate and treat the donor during the organharvesting period.

Since the development of the Th1/Th2 paradigm of CD4 $\mathrm{T}$ cell subsets, it has become apparent that additional important subsets participate in immune responses including graft rejection. In particular, there has been an explosion in studies investigating the role of the Th17 and Treg subsets. As reviewed by Shilling and Wilkes in "Role of Th17 cells and IL-17 in Lung Transplant Rejection", Th17 cells, which have been implicated in autoimmune diseases like rheumatoid arthritis and multiple sclerosis, also plan an important role in host defense and mucosal immunity. Recent data have implicated Th17 cells and IL-17 in chronic rejection after lung transplantation in both animal models and humans. Whereas Th17 cells promote rejection, Treg cells can suppress immune responses including graft rejection. As reviewed by Neujahr and Larsen in "Regulatory T Cells in Lung Transplantation-an Emerging Concept" the regulation, or suppression, or alloimmunity can maintain graft survival. It has been proposed that the ratio of Treg/Th17 cells determines the phenotype of the immune response. Thus, manipulation of Treg cells provides potential novel therapeutic targets.

It is apparent that clinical tolerance (and conversely graft rejection) is complex involving multiple cells types and multiple mechanisms. For example, known mechanisms include clonal deletion, anergy, suppression (e.g. by Treg cells), antigen presentation and accommodation. In addition, the induction of tolerance likely involves both the innate and adaptive immune responses. To address the complexity of these responses, powerful approaches such as "omics" technologies analysing biological responses on a genome-wide scale are being applied as illustrated by the reviews of T. Mueller and the development of a signature of tolerance by M. Sarwal. As reviewed by P. Finn in "Lung Transplantation: Infection, Inflammation and the Microbiome", the metagenome of the lung may modulate the course of bronchiolitis obliterans syndrome. Studies of the metagenome facilitate the investigation of the complete ecosystem of the lung microbiota. To interpret the exploding quantities of data accruing from omics studies, and to develop biological understanding from the data, new systems biology approaches need to be employed, which are reviewed by myself in "Advances of Genomic Science and Systems Biology in Renal Transplantation". 